\section{The Flow of Blood through Narrow Tubes}

IN a recent letter, one of us ${ }^{1}$ hes shown that the flow of the blood of several species in a single capillary tube of radius $R$ and length $L$ follows wn equation rocently proposed by Casson ${ }^{2}$ for varnishes and inks.

$$
\left(\frac{P R}{2 L}\right)^{1 / 2}=k_{0}+k_{1}\left(\frac{4 V}{\pi R^{3}}\right)^{1 / 2}
$$

where $P$ is pressure, $V$ is volume flow/sec., $k_{0}$ and $k_{1}$ are constants. When $k_{0}$ becomes zero, this equation reduces to the well-known equation of Poiseuille, since the terms in parenthesis represent the stress and shear rate respectively, at the wall of the tube. When $k_{0}$ has finite values, however, it is a measure of a yield-value or critical shearing stress which, as pressure is raised, will be first reached at the capillary wall. As pressure still further increases, the critical distance $\left(r_{0}\right)$ from the centre at which this occurs, will steadily diminish. A similar phenomenon was studied many years ago for the Bingham equation, which differs from Casson's equation only in the absence of square roots; when Buckingham ${ }^{3}$ and, independently, Reiner ${ }^{4}$ evaluated the correct equation of flow for such a system

$$
V=\frac{\pi R^{4}}{8 k_{1}^{2} L}\left(P-\frac{4}{3} p+\frac{p^{4}}{P^{9}}\right)
$$

where $p$ is the pressure corresponding to the yieldvalue given by $p=2 L k_{0}{ }^{2} / R$.

In the present communication a similar treatment is applied to Casson's equation, since, like Bingham's equation, a correction to allow for the changing value of $r_{0}$ with increasing pressure must be made if a linear relation is to be obtained.

Re-writing equation (1) :

$$
k_{1} D^{1 / 2}=\tau^{1 / 2}-k_{0}
$$

where $\tau$ is the shear stress (absolute value). Integration gives:

$$
k_{1}{ }^{2} v=\frac{r \tau}{2}-\frac{4}{3} r \tau^{1 / 2} k_{0}+r k_{0}{ }^{2}+C
$$

where $v$ is the velocity of flow at distance $r$ from the centre of the tube. If the material adheres to the wall, $v=0$ when $r=R$, from which the integration constant $C$ can be calculated.

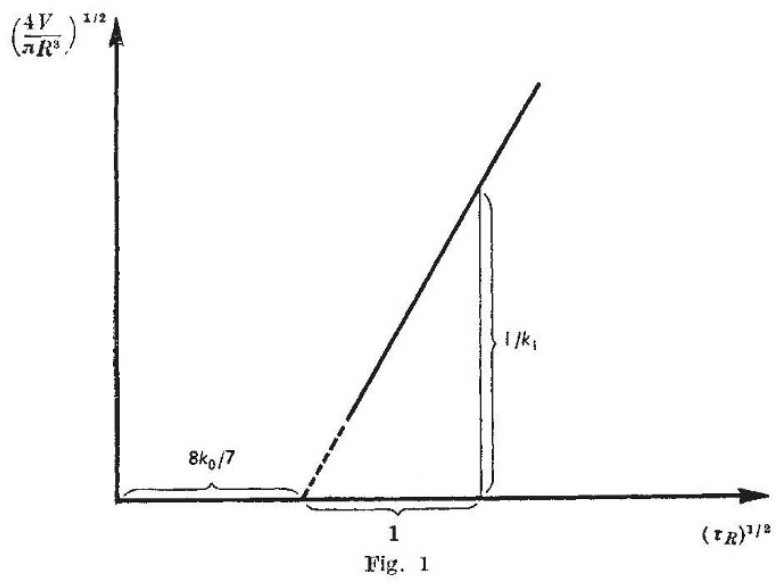

Introducing $\tau_{R}$ for the shear stress at the wall :

$V=\int_{0}^{R} r^{2} \mathrm{~d} v=\frac{\pi R^{3}}{4} \cdot \tau_{R}\left[1-\frac{16}{7} \frac{k_{0}}{\tau_{R}{ }^{1 / 2}}+\frac{4}{3} \frac{k_{0}^{2}}{\tau_{R}}-1 \frac{k_{0}{ }^{4}}{21}\right]$

This replaces the original Buckingham oquition(2). If $k_{0} / \tau_{R^{1 / 2}}$ is small : this approximates to

$$
V=\frac{\pi R^{3}}{4} \tau_{R}\left[1-\frac{8}{7} \frac{k_{0}}{\tau_{R}^{1 / 2}}\right]^{2}
$$

or :

$$
\left(\frac{4 V}{\pi R^{3}}\right)^{1 / 2}=\frac{1}{k_{1}}\left(\tau_{R^{1 / 2}}-\frac{8}{7} k_{0}\right) .
$$

Thus if $\left(\frac{4 V}{\pi R^{3}}\right)^{1 / 2}$ is plotted against $\tau_{R}{ }^{1 / 2}$, a straight line is obtained, from which $k_{1}$ and $k_{0}$ can be determined as shown in Fig. 1.

\section{ReINER}

G. W. SCOTt BLAIR*

Israel Institute of Technology, Haifa, Israel.

- Permanent address : National Institute for Research in Dairying, University of Reading.

${ }^{1}$ Scott Blair, G. W., Nature, 183, 613 (1959).

2 Casson, N., Chap. 5 of "The Rheology of Disperse Systems", ed. C. C. Mill. (Pergamon Press, London, 1959).

'Buckingham, E.' Proc. Amer. Soc. Tcst. Mater., 21, 1154 (1921).

4 Reiner, M., Kolloidzschr., 39, 80 (1926)

\section{Substrate Damage in Film Thickness Measurement by Beam Interferometry}

In the Tolansky method for the measurement of the thickness of thin films by multiple beam interference we have used a narrow channel in the film rather than a sharp step ${ }^{1}$. The channel can be formed by gently drawing a needle across the film before the deposition of the reflecting over-layer. The technique is easily applied and hes several advantages especially when the substrate is not optically flat as in the case of microscope slides.

Weaver and Benjamin ${ }^{2}$ have recently directed attention to a possible source of error in the technique.

They report that in order to form clear channels through films, deformations of the glass substrate by the needle can occur. For chromium films they measured deformations amounting to several hundred angstroms. Unfortunately no indication was given as to the nature and shape of the deformation.

We have found that while it is certainly possible to mar the glass with a steel needle, any damage can be detected by the irregularities in the shape of the fringe. Hence appreciable errors in thickness arising from damage to the substrate can be avoided.

Dr. Weaver kindly sent us some of the gramophone needles which they use, and we have compared the scratches made by these needles with the scratches made by the sewing needles which we often employ. The results are shown in Fig. 1, which illustrates fringes at a low order of interference ${ }^{3}$. Fringes $(a)$ are from scratches through a film made with a sewing needle held at about $45^{\circ}$ to the direction of motion.

From (i) to (iv) the loads increase in the range 5-200 gm. Fringes $(b i-i v)$ are from scratches made with a steel gramophone needle. It is evident that 